\title{
Hot origin of the Little Bang
}

\author{
S.V. Akkelin ${ }^{1,2,3}$ \\ ${ }^{1}$ Bogolyubov Institute for Theoretical Physics, \\ Metrolohichna 14b, 03680 Kiev, Ukraine \\ 2 Institut für Theoretische Physik, Universität Heidelberg, \\ Philosophenweg 16, 69120 Heidelberg, Germany \\ 3 ExtreMe Matter Institute EMMI, \\ GSI Helmholtzzentrum für Schwerionenforschung, \\ Planckstraße 1, 64291 Darmstadt, Germany
}

\begin{abstract}
Ultrarelativistic heavy ion collisions produce a quark-gluon matter which lies in the future light cone originating from given points on the $t=z=0$ plane of the Minkowski spacetime manifold. We show that in a weak coupling regime the Minkowski vacuum of massless fields presents itself in the "Little Bang" region as a thermal state of low $p_{T}$ particles, in close analogy to the Unruh effect for uniformly accelerated observers which are causally restricted to a Rindler wedge. It can shed some light on the mechanisms of early time thermalization in ultrarelativistic heavy ion collisions.
\end{abstract}

PACS numbers: 25.75.-q,03.70.+k 


\section{INTRODUCTION}

The "Little Bang" made in an ultrarelativistic heavy ion collision of the two Lorentz contracted nuclei starts from the spacetime region near $t \simeq z \simeq 0$ (in the laboratory frame), and produces initially very dense quark-gluon matter which then expands (mostly in the longitudinal direction) and eventually undergoes a transition to hadronic degrees of freedom (for recent reviews see, e.g., Refs. [1-3] and references therein). It is firmly established now that the quark-gluon matter created in these collisions seems to behave like a fluid [4-7] on very short time scales, but a first principle proof of the applicability of hydrodynamics for the initially far-from-equilibrium quark-gluon plasma is still lacking (for first-principles description of the early-time dynamics in ultrarelativistic heavy ion collisions see, e.g., Refs. [8, 9]) even though considerable progress has been made in the past years (for recent reviews see, e.g., Refs. [10 12] and references therein). There are many similarities (as well as differences) between the physics of Little Bang fireballs created in ultrarelativistic heavy ion collisions, and the cosmology (see, e.g., Refs. [13, 14]). Even widely utilized $t, z$ parametrization of spacetime region occupied by the expanded fireball [15] is nothing but the Milne coordinates [16, 17] which are, in fact, merely two-dimensional coordinate transformations of corresponding (future light cone) causally-connected region of the flat (Minkowski) spacetime manifold.

If one take such a Milne "universe" (future light cone) as a spacetime in its own right, then one can define vacuum state (or states) in the corresponding spacetime region. Indeed, it is well known (see, e.g., Ref. [16]) that solution of the free Klein-Gordon equation for massive scalar field in the two-dimensional Milne universe can be written in terms of either Bessel or Hankel functions, from which two complete sets of normalized modes, that are related by the Bogolyubov transformation, can be constructed. One of the pure vacuum states (associated with the Hankel functions) is, in fact, analogous to the usual Minkowski vacuum which is the ground state with respect to the Hamiltonian that is generator of the time translations. This vacuum is ill-defined in the massless limit for zero transverse

momenta (then $m_{T}=\sqrt{m^{2}+p_{T}^{2}}=0$ ), as well as initially, at $t=0$, for any $m_{T}$. The other one (associated with the Bessel functions) is analogous to the Rindler vacuum of an uniformly accelerated observer. There is thermal-like relation between these vacuum states, and the corresponding temperature is inversely proportional to the time, see details in Ref. [16]. 
In the present article we take a different approach. Namely, we assume that unlike true Milne universe, which is a spacetime in its own right, the spacetime region occupied by the matter produced in an ultrarelativistic heavy ion collision is embedded into the larger Minkowski spacetime with corresponding (Minkowski) vacuum state. Then the question arises: how does a "relevant" restriction of the Minkowski vacuum state to the Milne spacetime subspace look like for corresponding (local) operators which belong to this subspace ?1

The problem of how the Minkowski vacuum state looks like for an observer restricted to some causally-connected spacetime region is not new and was discussed earlier. A well known example is the Unruh effect [18] (for reviews see, e.g., Refs. [16, 19, 20], and for discussions about the validity of the Unruh effect see Refs. [21 23]) which is the result of a restriction of an uniformly accelerated observer to a Rindler wedge whose borderlines correspond to the event horizon. It was shown that the Minkowski vacuum expectation values of the local field operators restricted to this wedge seem to be calculated in an impure thermal-like state with respect to the wedge preserving generator of the corresponding time-like translations, and this generator is proportional to a Hamiltonian that is associated to a proper time of an uniformly accelerated (and, thereby, "eternal") observer.2 The corresponding "temperature" is proportional to an constant proper acceleration of an uniformly accelerated observer. For free fields, it was demonstrated that the Unruh effect follows from the fact that the Minkowski vacuum state in the Rindler basis can be written as an entangled state between two sets of modes, respectively spanning left and right Rindler wedges [18 20, 26]. Then, accounting that an uniformly accelerated observer is constrained to move in one of the Rindler wedges, one gets that the reduced density matrix corresponds to a thermal state with the Unruh temperature proportional to the observer's acceleration. Interestingly, a similar result was recently obtained in Ref. [27] for two-dimensional scalar field quantized in the Milne coordinates. Namely, it was shown that between the massless free fields within the future and past light cone there is the same entanglement as for fields between the left and right Rindler wedges, and the existence of a vacuum thermal effect for an inertial observer

1 Notice that unlike the classical field theory, where vacuum really means an empty space, the quantum field vacuum is a pure state which contains nontrivial space-like quantum correlations. The reason is the following: while the commutator of field operators does vanish when $x$ and $y$ are space-like related, the expectation value $<0|\phi(x) \phi(y)+\phi(y) \phi(x)| 0>$ is not equal to $2<0|\phi(x)| 0><0|\phi(y)| 0>$, it is a manifestation of space-like quantum correlations (entanglement) in the vacuum.

${ }^{2}$ For analysis of cases where an observer undergoes 3 non-uniform acceleration see e.g. Refs. [24, 25]. 
constrained to interact with the field in only the future or the past light cone was noted. It is also worth noting that in Ref. [28] the Unruh effect was studied for an observer with a finite lifetime, who has access to the local observables associated to a finite spacetime region called a "diamond". Using the thermal time hypothesis, it was shown that the Unruh effect exists for such an observer too, and that corresponding temperature is time-dependent and does not vanish even in the limit in which the acceleration is zero.

In our opinion, the possible existence of an analogue of the Unruh effect in the Milne "universe" (future light cone) appears worthy of further investigation, especially in respect to the longstanding problem of early time thermalization in relativistic heavy ion collisions, see e.g. Refs. [1, 2, 10, 11] 3 In the present article, we demonstrate that in a weak coupling approximation of effectively two-dimensional massless scalar field model the Minkowski vacuum state looks like the mixed thermal state for operators restricted to the future light cone with the corresponding time-like generator as the Hamiltonian, an analogue to the Unruh effect. We obtain our results using the method which was early applied in Ref. [36] to derive the Unruh effect for scalar field with interactions.

\section{MINKOWSKI VACUUM AND RELATIVISTIC HEAVY ION COLLISIONS}

Most of the particles detected in an ultrarelativistic heavy ion central collision are produced by the relevant subsystem which is created in the future light cone with the beginning at the $t=z=0$ plane (more exactly, at spacetime region near $t \simeq z \simeq 0$ ), where two Lorentz-contracted nuclei collide and interact. The (relevant) past evolution of this subsystem is encoded into the corresponding initial state within the future light cone. If one defines an initial state at $\tau=\sqrt{t^{2}-z^{2}}=$ const hypersurface, then such a hypersurface encloses a spacetime region within the future light cone. Separation of such a subsystem means that one needs trace over unobservables (e.g., correlations with irrelevant degrees of freedom outside the future light cone). Similar to quantum mechanics, such a trace-out procedure can result in loss of an information. It is worth noting that quantum field theory is in a certain sense more "quantum" than quantum mechanics because presence of the quantum vacuum in the former. In quantum field theory, unlike of quantum mechanics, there

3 The Unruh effect has been considered as a possible explanation of the observed thermal behavior in relativistic heavy ion collisions in Refs. 29 35], where the dynamical origin of the (transient) acceleration is related with the strength of the color field. 
are vacuum correlations (entanglements) between quantum fields which are localized inside the region enclosed by the hypersurface $\tau=$ const, and quantum fields localized outside the one. Therefore, one can expect that under certain conditions the Minkowski vacuum can look like (time-dependent) thermal mixed state for quantum operators depending on the values of quantum field in spacetime points belonging only the region enclosed by the $\tau=$ const hypersurface. In such a case the expectation values of corresponding operators in the Minkowski vacuum state can coincide with ones calculated in some thermal-like state just due to the entanglement of quantum vacuum fluctuations.

To demonstrate how this idea works, let us consider a massless scalar quantum field model with a classical action

$$
S=\int d t d^{3} r\left[\frac{1}{2}\left(\frac{\partial \phi}{\partial t}\right)^{2}-\frac{1}{2}\left(\frac{\partial \phi}{\partial \mathbf{r}}\right)^{2}-V(\phi)\right]=\int d t d^{3} r L
$$

where $\mathbf{r}=(x, y, z), V(\phi)$ is a polynomial function of $\phi$, and $L$ is the corresponding Lagrangian density in the global Minkowski spacetime.

It is well known that in the path-integral formulation of quantum field theory the transition amplitude from the initial vacuum $\mid 0$, in $\rangle$ to the final vacuum $\mid 0$, out $\rangle$ in the presence of a source $J$ is given by the generating functional ( $N$ is a normalization factor)

$$
Z[J]=\langle 0, \text { out } \| 0, \text { in }\rangle=N \int D \phi \exp i(S(\phi)+J \phi),
$$

and we use the shorthand symbol $J \phi$ for four-dimensional integral over product of external source and $\phi$. The functional integration is taken over the space of all possible functional forms of $\phi$ with some initial and final boundary values.

The expectation values of field operators in the Heisenberg picture can be obtained by functional differentiations with respect to the external classical source $J$. If $J=0$, these two vacua coincide and reduce to the time-translation invariant vacuum $|0\rangle$. Then initial and final boundary $\phi$ values coincide, and

$$
Z \equiv Z[0]=\langle 0|| 0\rangle=\left\langle 0\left|e^{-i H^{[t]}\left(t_{\text {out }}-t_{\text {in }}\right)}\right| 0\right\rangle=N \int D \phi \exp (i S(\phi))
$$

becomes the vacuum to vacuum transition amplitude. Here $H^{[t]}$ is the corresponding Hamiltonian, the superscript $[t]$ means that the Hamiltonian $H^{[t]}$ is the generator of the time translation in the flat Minkowski spacetime.

Let us recall that one can regard $Z$ as the partition function for a thermal system at zero temperature (see, e.g., Refs. [37-39]) with respect to the Hamiltonian $H^{[t]}$. With this aim 
in view let us assume that $t_{\text {out }}=-t_{\text {in }}=\infty$. Then, by making the time pure imaginary (so called Euclidian time), $i t=t_{E}, t_{E}$ is real, we get the Euclidian action

$$
S_{E}=\int d t_{E} d^{3} r\left[\frac{1}{2}\left(\frac{\partial \phi}{\partial t_{E}}\right)^{2}+\frac{1}{2}\left(\frac{\partial \phi}{\partial \mathbf{r}}\right)^{2}+V(\phi)\right]=-\int d t_{E} d^{3} r L\left(\phi, i \frac{\partial \phi}{\partial t_{E}}\right) .
$$

Now, let integration goes over all periodic paths that have the same classical $\phi$ values at $t_{E}=-\infty$ as at $t_{E}=\infty$. Then the Euclidian functional,

$$
Z_{E}=N_{E} \int_{\text {periodic }} D \phi \exp \left(-S_{E}\right)
$$

where $N_{E}$ is a normalization factor, is equivalent to the thermal partition function $\operatorname{Tr}\left[e^{-\beta H^{[t]}}\right]$ at zero temperature, $T^{-1}=\beta \rightarrow \infty$. Indeed, one can see that

$$
Z_{E}=\lim _{\beta \rightarrow \infty} \operatorname{Tr}\left[|0\rangle\langle 0| e^{-\beta E_{0}}\right]
$$

and if we assume that the energy of the Minkowski vacuum, $E_{0}$, is zero (it is possible if the spectrum of the Hamiltonian is bounded from below), we finally get

$$
Z_{E}=Z=\langle 0|| 0\rangle
$$

This allows one to consider the usual vacuum as a zero temperature thermal state, and Eq. (5) as the Euclidean functional integral representation of the Minkowski vacuum.

It is important to note that a global vacuum state of the field at zero temperature in the complete space can turn into a thermal state with non-zero temperature in the incomplete space [36]. It can easily be seen using polar variables,

$$
\begin{aligned}
t_{E} & =a^{-1} e^{a \eta} \sin a \zeta, \\
z & =a^{-1} e^{a \eta} \cos a \zeta,
\end{aligned}
$$

where $a$ is a scale-parameter, and performing change of integration variables in Eq. (4). Then we get

$$
S_{E}=\int_{0}^{\beta_{R}} d \zeta \int d^{2} r_{T} d \eta\left[\frac{1}{2}\left(\frac{\partial \phi}{\partial \zeta}\right)^{2}+\frac{1}{2}\left(\frac{\partial \phi}{\partial \eta}\right)^{2}+e^{2 a \eta}\left(\frac{1}{2}\left(\frac{\partial \phi}{\partial \mathbf{r}_{T}}\right)^{2}+V(\phi)\right)\right]
$$

where

$$
\beta_{R}=\frac{2 \pi}{a}
$$


in order to cover the whole Euclidian $\left(t_{E}, \mathbf{r}_{T}, z\right)$ space, and we allow $\int D \phi$ to go over all periodic paths. One can see that the Euclidian functional integral (5), (10), (11) is just a representation of the thermal partition function with periodic boundary conditions $\phi(\zeta=$ $0)=\phi\left(\zeta=\beta_{R}\right)$ with respect to imaginary "time" $\xi=i \zeta, \zeta$ is real,

$$
Z=\operatorname{Tr}\left[e^{-\beta_{R} H^{[\xi]}}\right]
$$

where the "Hamiltonian" $H^{[\xi]}$ is the generator of translations in the time-like direction with respect to real $\xi$. Note that the thermal bath refers to the Hamiltonian $H^{[\xi]}$ which is different from the Hamiltonian $H^{[t]}$ whose lowest energy eigenstate defines the Minkowski vacuum.

Now, let us recall how the Unruh effect is related with the above formal approach (for details see Refs. [20, 36]). We begin with introducing the so-called Rindler coordinates in the Minkowski spacetime. Namely, the flat spacetime expressed in the globally defined Minkowski coordinates $(t, x, y, z)$ can be divided by lines $t= \pm z$ into four quadrants which we call as future $(t>|z|)$ and past $(-t>|z|)$ light cones, and the right $(z>|t|)$ and left $(-z>|t|)$ Rindler wedges. Rindler coordinates $(\xi, \eta)$ are related with Minkowski coordinates $(t, z)$ in the right Rindler wedge as

$$
\begin{gathered}
z=a^{-1} e^{a \eta} \cosh a \xi \\
t=a^{-1} e^{a \eta} \sinh a \xi
\end{gathered}
$$

Notice that the $(-\infty,+\infty)$ region of $\xi, \eta$ coordinates covers the right Rindler wedge, the two other coordinates $\mathbf{r}_{T}=(x, y)$ are the same both in the Minkowski and Rindler frames. The trajectory defined by constant values of $\eta$ and $\mathbf{r}_{T}$ describes the motion of an observer with constant proper acceleration $a e^{-a \eta}>04$ Using (13), (14), the Minkowski line element restricted to the Rindler wedge becomes

$$
d s^{2}=d t^{2}-d \mathbf{r}_{T}^{2}-d z^{2}=e^{2 a \eta}\left(d \xi^{2}-d \eta^{2}\right)-d \mathbf{r}_{T}^{2}
$$

One can see that $e^{a \eta} \xi$ is a proper time of the uniformly accelerated Rindler observer, and the Rindler time-like coordinate $\xi$ is a measure of a proper time along the trajectory with $\eta=0$.

\footnotetext{
${ }^{4}$ If $\left(\xi, \mathbf{r}_{T}, \eta\right)$ is treated not as a physical frame of reference but as an abstract non-inertial coordinate system, then such an observer is an hypothetical one.
} 
To define the Lagrangian density in the Rindler coordinates, notice that the action (1) for the scalar field with potential $V(\phi)$ can be rewritten in the Rindler frame (13), (14) as

$$
\begin{array}{r}
S=\int d \xi d \eta d^{2} r_{T}\left[\frac{1}{2}\left(\frac{\partial \phi}{\partial \xi}\right)^{2}-\frac{1}{2}\left(\frac{\partial \phi}{\partial \eta}\right)^{2}-e^{2 a \eta}\left(\frac{1}{2}\left(\frac{\partial \phi}{\partial \mathbf{r}_{T}}\right)^{2}+V(\phi)\right)\right] \equiv \\
\int d \xi d \eta d^{2} r_{T} L_{R},
\end{array}
$$

where $L_{R}$ is the corresponding Lagrangian density, and we take into account that

$$
d t d z=d \eta d \xi e^{2 a \eta} .
$$

The corresponding Hamiltonian density, $H_{R}$, then reads

$$
H_{R}=\Pi^{[\xi]} \frac{\partial \phi}{\partial \xi}-L_{R}=\frac{1}{2}\left(\frac{\partial \phi}{\partial \xi}\right)^{2}+\frac{1}{2}\left(\frac{\partial \phi}{\partial \eta}\right)^{2}+e^{2 a \eta}\left(\frac{1}{2}\left(\frac{\partial \phi}{\partial \mathbf{r}_{T}}\right)^{2}+V(\phi)\right)
$$

where $\Pi^{[\xi]}=\frac{\partial \phi}{\partial \xi}$ is the conjugated field momentum.

The well known equivalence in the Rindler wedge between the Minkowski vacuum partition function, $Z=\langle 0 \| 0\rangle$, and thermal partition function,

$$
Z_{R}=\operatorname{Tr}\left[e^{-\beta_{R} H^{[\xi]}}\right],
$$

defined by the Rindler Hamiltonian $H^{[\xi]}=\int d \eta d^{2} r_{T} H_{R}$ which generates evolution along $\xi$ in Minkowski spacetime, can easily be seen using an Euclidean functional integral representation of the thermal partition function (20):

$$
Z_{R}=N_{E} \int D \phi \exp \left[\int_{0}^{\beta_{R}} d \zeta \int d^{2} r_{T} d \eta L_{R}\left(\phi, i \frac{\partial \phi}{\partial \zeta}\right)\right],
$$

with periodic boundary conditions. Now, to derive the Unruh effect, notice that substitution of the imaginary "time" $\xi=i \zeta, \zeta$ is real, in Eqs. (13), (14) results in Eqs. (8) and (9).

Then, performing a corresponding change of integration variables in Eq. (21), and assuming that

$$
\beta_{R}=\frac{2 \pi}{a}
$$

in order to cover the whole Euclidian $\left(t_{E}, \mathbf{r}_{T}, z\right)$ space, we get that the thermal partition function in the Rindler wedge lying to one side of an infinite $x y$ plane, $Z_{R}$, can be expressed as the partition function of the Minkowski vacuum state, $Z$,

$$
\operatorname{Tr}\left[e^{-\frac{2 \pi}{a} H^{[\xi]}}\right]=\langle 0|| 0\rangle
$$


with Unruh temperature that is proportional to a constant proper acceleration of an uniformly accelerated observer,

$$
T_{R}=\beta_{R}^{-1}=\frac{a}{2 \pi}
$$

Equality (23) means that the expectation value in the Minkowski vacuum state of any operators that are causally bounded (restricted) to the (right) Rindler wedge is equivalent to a thermal average at the constant Unruh temperature [36]:

$$
\left\langle 0\left|T_{t}\left(\phi\left(x_{1}\right) \phi\left(x_{2}\right) \ldots\right)\right| 0\right\rangle=\frac{\operatorname{Tr}\left[e^{-\beta_{R} H^{[\xi]}} T_{\xi}\left(\phi\left(x_{1}\left(\xi_{1}, \mathbf{r}_{T 1}, \eta_{1}\right)\right) \phi\left(x_{2}\left(\xi_{2}, \mathbf{r}_{T 2}, \eta_{2}\right)\right) \ldots\right)\right]}{\operatorname{Tr}\left[e^{-\beta_{R} H^{[\xi]}}\right]}
$$

where $T_{t}$ and $T_{\xi}$ denote time and $\xi$ ordering, respectively, and $x_{i}\left(\xi_{i}, \mathbf{r}_{T i}, \eta_{i}\right)$ represents the same spacetime point as $x_{i}$ but in the Rindler coordinates. Then for an uniformly accelerated observer the Minkowski vacuum is seen as a thermal bath with temperature proportional to the magnitude of the acceleration.

At this point one may wonder how an energy conservation is maintained. To see how it proceeds, note that the left-hand-side of Eq. (25) is defined with respect to the Minkowski vacuum, while the right-hand-side is defined with respect to the Rindler vacuum 5 Their energy densities are different, as one can see by calculating expectation values of the stresstensor $T_{\mu \nu}$ [16]. Namely, if we accept that the energy density of the Minkowski vacuum is equal to zero, then the energy density of the Rindler vacuum is negative. The thermal state of the Rindler quanta increases energy density from negative till zero value and compensates this difference to maintain energy conservation.

Now, let us consider the future light cone and associate the Milne frame with the system of the (hypothetical) observers which move with different but constant longitudinal velocities in such a way that their world lines begin at $z=t=0$. In the Milne frame, coordinates $(\xi, \eta)$ are related with Minkowski coordinates as:

$$
\begin{aligned}
& t^{\prime}=b^{-1} e^{b \xi} \cosh b \eta \\
& z^{\prime}=b^{-1} e^{b \xi} \sinh b \eta
\end{aligned}
$$

where primes are introduced to distinguish the parametrization of the Minkowski coordinates in the future light cone from the parametrization (13), (14) in the right Rindler wedge. Here

\footnotetext{
${ }^{5}$ The concept of vacuum is observer dependent: An uniformly accelerated observer determines a zero energy state with respect to $H^{[\xi]}$.
} 
$b$ is a scale-parameter, and the two other coordinates $\mathbf{r}_{T}=(x, y)$ are the same both in the Minkowski and Milne frames. One can see that the $(-\infty,+\infty)$ region of $\xi, \eta$ coordinates covers the whole future light cone. Taking into account (26) and (27) we can calculate the Minkowski line element restricted to the future light cone and get

$$
d s^{2}=d t^{\prime 2}-d \mathbf{r}_{T}^{2}-d z^{\prime 2}=e^{2 b \xi}\left(d \xi^{2}-d \eta^{2}\right)-d \mathbf{r}_{T}^{2}
$$

It follows from Eq. (28) that the Minkowski metrics is non-static with respect to $\xi$. It is convenient to introduce dimensionless variables $\bar{\eta}, \bar{\xi}$ :

$$
\begin{gathered}
\bar{\eta}=b \eta \\
\bar{\xi}=b \xi
\end{gathered}
$$

and define

$$
\tau=b^{-1} e^{\bar{\xi}}
$$

Then

$$
\begin{aligned}
t^{\prime} & =\tau \cosh \bar{\eta} \\
z^{\prime} & =\tau \sinh \bar{\eta}
\end{aligned}
$$

and one can see that $\tau$ is a proper time of an inertial Milne observer with constant rapidity $\bar{\eta}$ and constant transverse coordinates $\mathbf{r}_{T}$, because

$$
d s^{2}=d t^{\prime 2}-d \mathbf{r}_{T}^{2}-d z^{\prime 2}=d \tau^{2}-\tau^{2} d \bar{\eta}^{2}-d \mathbf{r}_{T}^{2}
$$

To define Lagrangian density in the future light cone in the coordinates $(\xi, \eta)$, one can rewrite action (1) in the Milne frame (26), (27) as

$$
\begin{array}{r}
S=\int d \xi d \eta d^{2} r_{T}\left[\frac{1}{2}\left(\frac{\partial \phi}{\partial \xi}\right)^{2}-\frac{1}{2}\left(\frac{\partial \phi}{\partial \eta}\right)^{2}-e^{2 b \xi}\left(\frac{1}{2}\left(\frac{\partial \phi}{\partial \mathbf{r}_{T}}\right)^{2}+V(\phi)\right)\right] \equiv \\
\int d \xi d \eta d^{2} r_{T} L_{M},
\end{array}
$$

where $L_{M}$ is the corresponding Lagrangian density, and we take into account that

$$
d t d z=d \eta d \xi e^{2 b \xi}
$$

The Hamiltonian density, $H_{M}$, is

$$
H_{M}=\frac{1}{2}\left(\frac{\partial \phi}{\partial \xi}\right)^{2}+\frac{1}{2}\left(\frac{\partial \phi}{\partial \eta}\right)^{2}+e^{2 b \xi}\left(\frac{1}{2}\left(\frac{\partial \phi}{\partial \mathbf{r}_{T}}\right)^{2}+V(\phi)\right)
$$


and the corresponding Hamiltonian that generates translation in the time-like direction with respect to $\xi$ is $H^{[\xi]}=\int d \eta d^{2} r_{T} H_{M}$. Notice that unlike the Hamiltonian of an uniformly accelerated observer in the Rindler wedge, $H^{[\xi]}$ is explicitly time-dependent ( $\xi$ is the timelike parameter in the future light cone). To proceed further, let us assume that the term in the Hamiltonian with explicit $\xi$-dependence is small and can be neglected. To see under what conditions it is the case, let us rewrite action (35) in variables $\left(\tau, \mathbf{r}_{T}, \bar{\eta}\right)$, then

$$
\begin{array}{r}
S=\int d \tau d \bar{\eta} d^{2} r_{T}\left[\frac{\tau}{2}\left(\frac{\partial \phi}{\partial \tau}\right)^{2}-\frac{1}{2 \tau}\left(\frac{\partial \phi}{\partial \bar{\eta}}\right)^{2}-\tau\left(\frac{1}{2}\left(\frac{\partial \phi}{\partial \mathbf{r}_{T}}\right)^{2}+V(\phi)\right)\right] \equiv \\
\int d \tau d \bar{\eta} d^{2} r_{T} \bar{L}_{M},
\end{array}
$$

where $\bar{L}_{M}$ is the corresponding Lagrangian density. The corresponding Hamiltonian density, $\bar{H}_{M}$, reads

$$
\bar{H}_{M}=\frac{\tau}{2}\left(\frac{\partial \phi}{\partial \tau}\right)^{2}+\frac{1}{2 \tau}\left(\frac{\partial \phi}{\partial \bar{\eta}}\right)^{2}+\tau\left(\frac{1}{2}\left(\frac{\partial \phi}{\partial \mathbf{r}_{T}}\right)^{2}+V(\phi)\right) .
$$

The Hamiltonian that generates translation in the time-like direction with respect to $\tau$ is $H^{[\tau]}=\int d \bar{\eta} d^{2} r_{T} \bar{H}_{M}$. Note that

$$
H^{[\xi]}=b \tau H^{[\tau]}
$$

and that the dependence on the scale-parameter $b$ is canceled out in $H^{[\tau]}$. It follows from (41) that the last term in $\bar{H}_{M}$ is small and can be neglected if interactions are weak, and if the spacetime is effectively two-dimensional, the latter means that only modes with low transverse momenta, $p_{T}^{2} \tau^{2} \ll 1$, are considered 6

To find an expression for the vacuum partition function $Z=\langle 0|| 0\rangle$ in the future light cone, let us assume that the above mentioned conditions are satisfied, and hypothesize that the vacuum partition function, restricted on the future light cone, can be associated in the weak coupling approximation and for low $p_{T}$ particles with the thermal partition function $Z_{M}$

$$
Z_{M}=\operatorname{Tr}\left[e^{-\beta_{M} H^{[\xi]}}\right]=\operatorname{Tr}\left[e^{-\beta_{M} b \tau H^{[\tau]}}\right]
$$

where $\beta_{M}$ will be specified below.

\footnotetext{
${ }^{6}$ Also, one can notice from Eq. (41) that massive quantum field is effectively massless for very early times when $m \tau \ll 1$.
} 
To establish a relation in the future light cone between the vacuum partition function $Z=\langle 0|| 0\rangle$ and the thermal partition function $Z_{M}$, approximate first $Z_{M}$ as

$$
Z_{M} \simeq Z_{F M}=\operatorname{Tr}\left[e^{-\beta_{M} H_{F}^{[\xi]}}\right]
$$

where $H_{F}^{[\xi]}=\int d \eta d^{2} r_{T} H_{F M}$, and $H_{F M}$ is the Hamiltonian density of free massless field in the $(\xi, \eta)$ spacetime,

$$
H_{F M}=\frac{1}{2}\left(\frac{\partial \phi}{\partial \xi}\right)^{2}+\frac{1}{2}\left(\frac{\partial \phi}{\partial \eta}\right)^{2}
$$

Then, let us rewrite $Z_{F M}$ as the Euclidian functional integral,

$$
\begin{array}{r}
Z_{F M}=N_{F E} \int D \phi \exp \left[\int_{0}^{\beta_{M}} d \zeta \int d^{2} r_{T} d \eta L_{F M}\left(\phi, i \frac{\partial \phi}{\partial \zeta}\right)\right]= \\
N_{F E} \int D \phi \exp \left[-\int_{0}^{\beta_{M}} d \zeta \int d^{2} r_{T} d \eta\left(\frac{1}{2}\left(\frac{\partial \phi}{\partial \zeta}\right)^{2}+\frac{1}{2}\left(\frac{\partial \phi}{\partial \eta}\right)^{2}\right)\right]
\end{array}
$$

with periodic boundary conditions $\phi(\zeta=0)=\phi\left(\zeta=\beta_{M}\right)$ for arbitrary $\beta_{M}$ with respect to $\zeta$, here $\zeta$ is real and $\xi=i \zeta$.

Now, notice that for free massless field in effectively two-dimensional spacetime the functional form of the Euclidian functional integral (21) in the Rindler wedge non-inertial coordinate system is

$$
Z_{F R}=N_{F E} \int D \phi \exp \left[-\int_{0}^{\beta_{R}} d \zeta \int d^{2} r_{T} d \eta\left(\frac{1}{2}\left(\frac{\partial \phi}{\partial \zeta}\right)^{2}+\frac{1}{2}\left(\frac{\partial \phi}{\partial \eta}\right)^{2}\right)\right]
$$

and thereby is identical with functional form of the Euclidian functional integral (46) in the future light cone inertial coordinate system. Then, to establish relation of $Z_{F M}$ with the Minkowski vacuum partition function, one can apply the same method which is used for the thermal partition function $Z_{R}$ in the Rindler wedge. Namely, assuming that $\beta_{M}=2 \pi / b$ and changing integration variables 7

$$
\begin{array}{r}
t_{E}=b^{-1} e^{b \eta} \sin b \zeta, \\
z=b^{-1} e^{b \eta} \cos b \zeta
\end{array}
$$

in Eq. (46), we get that

$$
Z_{F M}=\langle 0 \| 0\rangle
$$

\footnotetext{
${ }^{7}$ It is worth emphasizing, to avoid misunderstanding, that this is just a change of integration variables but not coordinates.
} 
for $\beta_{M}=2 \pi / b$. Finally, assuming that $Z_{M} \simeq Z_{F M}$ and taking into account Eq. (43), we get that with respect to $H^{[\tau]}$ the pure Minkowski vacuum state looks like mixed thermal state with temperature $1 / 2 \pi \tau$,

$$
\langle 0|| 0\rangle \simeq \operatorname{Tr}\left[e^{-2 \pi \tau H^{[\tau]}}\right]
$$

Notice that the value of the auxiliary scale-parameter $b$ is arbitrary: correspondence with the Unruh effect is reached for any $b$, and the dependence on the scale-parameter $b$ is canceled out in variables $\left(\tau, \mathbf{r}_{T}, \bar{\eta}\right)$. Equality (51) means that the expectation value in the Minkowski vacuum state of quantum operators depending on the values of the quantum field in spacetime points belonging only to the region inside the future light cone is approximately equivalent to a thermal average at the temperature $1 / 2 \pi \tau$.

The corresponding thermal statistical operator in the right-hand-side of Eq. (51) is defined over the analog of the Rindler vacuum, that is one of the states in the expanding Milne universe [16, 20]. It is known that the difference between expectation values of the energymomentum tensor of massless fields calculated in the analog of the Rindler vacuum and in the Minkowski vacuum is negative, time-dependent and tends to zero for asymptotic times [16]. Then, if we accept that the energy density of the Minkowski vacuum is equal to zero, the energy density of the analog of the Rindler vacuum is negative, and, by correspondence to the Unruh effect, the thermal state in the right-hand-side of Eq. (51) increases energy density from negative till zero value.

Finally, let us consider our findings in view of relativistic heavy ion collisions and estimate the relevant parameters. First, recall that in the weak coupling limit of the QCD (corresponding to the high energy limit $\sqrt{s} \rightarrow \infty$ of collisions of heavy nuclei) the initial conditions of nuclear collisions are fairly well understood in terms of the Color Glass Condensate framework [40], that is the effective field theory which describes universal properties of saturated gluons in wave functions of colliding nuclei. Saturation is characterized by a transverse momentum scale $Q_{s}$, typical values are estimated to be $Q_{s}^{2} \simeq 2 \mathrm{GeV}^{2}$ at $\mathrm{RHIC}$ and $Q_{s}^{2} \simeq 5 \mathrm{GeV}^{2}$ at the LHC [41]. Then, in the idealized high-energy limit of heavy ion collisions, the dynamics of the system right after the collision (at $\tau \ll Q_{s}^{-1}$ ) is that of over-occupied far-from-equilibrium gluon fields expanding in the longitudinal direction, with typical momentum $Q_{s}$ and a weak gauge coupling $\alpha_{s}\left(Q_{s}\right)$, usually referred to as the Glasma [42]. Because of the over-occupation, the system is initially strongly interacting even though 
the coupling is weak. At very early times $\tau \ll Q_{s}^{-1}$, the dynamics of the nonequilibrium Glasma created in such a collision is described in the midrapidity region with approximately boost invariant classical gauge fields screened on transverse distance scales $1 / Q_{s}$, rather than with particles (gluons). The classical fields are decayed (i.e., field expectation value becomes to be zero) and gluons are freed in a timescale $\tau \sim Q_{s}^{-1}$, see e.g. Refs. [8, 9]. One can expect that our results can be applicable after the decay of the classical gluon fields, i.e., at a lower bound of about $\tau_{\text {in }} \simeq Q_{s}^{-1}$, when dynamics becomes governed by the quantum vacuum and its excitations. It corresponds to a temperature $T_{i n}=1 / 2 \pi \tau_{i n} \simeq Q_{s} / 2 \pi$ of the Minkowski vacuum thermal bath 8 At the LHC, one can estimate $\tau_{\text {in }} \simeq 0.1 \mathrm{fm}$ and $T_{\text {in }} \simeq 0.35 \mathrm{GeV}$. Notice here, to avoid misunderstanding, that the latter is not the temperature of the whole quark-gluon system at $\tau_{\text {in }} \simeq 0.1 \mathrm{fm}$; in fact, $T_{i n} \simeq 0.35 \mathrm{GeV}$ is the temperature of the Minkowski vacuum thermal bath only.

Thermalization mechanism in relativistic heavy ion collisions is not yet fully understood. In the bottom-up thermalization scenario [43], for example, the pre-equilibrium evolution after decay of the classical color fields is divided into three temporal stages: (i) the system is dominated by the over-occupied hard gluons whose typical transverse momentum is $Q_{s}$; (ii) soft gluons are produced by collinear splitting processes; (iii) soft gluons thermalize first and form the thermal bath, then the thermal bath drains the energy from the hard gluons and make them thermalized. Therefore, in such a scenario thermalization proceeds from bottom to top in the energy scale. The approach which takes into account thermal-like properties of the Minkowski vacuum in the future light cone of relativistic heavy ion collisions suggests modification of the bottom-up thermalization scenario. Namely, because the hard gluons whose typical transverse momentum is $Q_{s}$ are immersed in the Minkowski vacuum thermal bath with the temperature $T=1 / 2 \pi \tau$, they start to thermalize already at the stage (i), immediately after they are freed from classical fields at $\tau \simeq Q_{s}^{-1}$.

Evidently, in the course of the system evolution weak coupling approximation becomes not valid. Then, strictly speaking, Eq. (51) is applicable for very early proper times only. However, even at later proper times one can expect that the pure Minkowski vacuum state looks like a mixed state with some thermal-like properties with respect to $H^{[\tau]}$. With increase

8 It is worth noting that the same expression for temperature was proposed in Refs. 29 31] for partons moving in strong color field with typical (transient) acceleration $\sim Q_{s}$ in analogy to the Unruh effect. Notice, however, that our approach here is quite different and is not based on a picture of accelerated partons at all. 
of $\tau$ the differences between the pure ground (vacuum) state of the Hamiltonian $H^{[\tau]}$ and the Minkowski vacuum state decrease resulting in gradual disappearance of the thermallike properties of the Minkowski vacuum with respect to the generator of the time-like translations in the future light cone, $H^{[\tau]}$.

\section{CONCLUSIONS}

We have studied how the global pure vacuum state in the Minkowski spacetime looks like for the Milne set of inertial observers that are locally restricted to the light cone with beginning at $t=z=0$. We found that in a weak coupling approximation of effectively twodimensional massless scalar field model a pure Minkowski vacuum state looks like the mixed thermal state with the Hamiltonian $H^{[\tau]}$ which is the generator of translations in the timelike direction with respect to (longitudinal) proper time $\tau=\sqrt{t^{2}-z^{2}}$, and whose lowest energy eigenstate does not coincide with the Minkowski vacuum. Effective spacetime twodimensionality for such a system means that we consider only $p_{T} \tau \ll 1$ modes (particles). We found that temperature of the corresponding thermal state varies with respect to proper time $\tau$ as $1 / 2 \pi \tau$. In other words, the Minkowski vacuum expectation value of the appropriate local quantum operators in the light cone can be interpreted in terms of a thermal-like mixture of states which differ locally from the Minkowski vacuum, an analogue of the Unruh effect.

In a relativistic nucleus-nucleus collision the abstract Milne coordinate system becomes physical reference frame, and our analysis suggests that created at $\tau \simeq Q_{s}^{-1}$ in the future light cone of a relativistic heavy ion collision quarks and gluons feel quantum fluctuations of the Minkowski vacuum as a quantum thermal bath of weakly interacting on-mass-shell soft gluons. That would speed up the process of "hydrodynamization" and, perhaps, can be responsible for the "direct photon flow puzzle" observed in ultrarelativistic heavy ion collisions [44, 45].

\section{ACKNOWLEDGMENTS}

I am grateful to J. Berges, K. Boguslavski, and S. Floerchinger for discussions. This research was supported in part by the ExtreMe Matter Institute EMMI at the GSI 
Helmholtzzentrum für Schwerionenforschung, Darmstadt, Germany.

[1] F. Gelis, Int. J. Mod. Phys. E 24, 1530008 (2015) [arXiv:1508.07974].

[2] K. Fukushima, Rep. Prog. Phys. 80, 022301 (2017) arXiv:1603.02340.

[3] P. Braun-Munzinger, V. Koch, T. Schäfer, J. Stachel, Phys. Rep. 621, 76 (2016) arXiv:1510.00442].

[4] U. Heinz, R. Snellings, Annu. Rev. Nucl. Part. Sci. 63, 123 (2013) arXiv:1301.2826.

[5] C. Gale, S. Jeon, B. Schenke, Int. J. Mod. Phys. A 28, 1340011 (2013) arXiv:1301.5893].

[6] P. Huovinen, Int. J. Mod. Phys. E 22, 1330029 (2013) arXiv:1311.1849].

[7] R. Derradi de Souza, T. Koide, T. Kodama, Prog. Part. Nucl. Phys. 86, 35 (2016) arXiv:1506.03863].

[8] J. Berges, K. Boguslavski, S. Schlichting, R. Venugopalan, Phys. Rev. D 89, 114007 (2014) arXiv:1311.3005].

[9] J. Berges, K. Boguslavski, S. Schlichting, R. Venugopalan, Phys. Rev. D 92, 096006 (2015) arXiv:1508.03073].

[10] A. Kurkela, Nucl. Phys. A 956, 136 (2016) arXiv:1601.03283].

[11] W. van der Schee, Nucl. Phys. A 967, 74 (2017) arXiv:1705.01556.

[12] P. Romatschke, Eur. Phys. J. C 77, 21 (2017) arXiv:1609.02820].

[13] U. Heinz, J. Phys.: Conf. Ser. 455, 012044 (2013) arXiv:1304.3634].

[14] S. Floerchinger, Nucl. Phys. A 956, 91 (2016) arXiv:1512.08388.

[15] J.D. Bjorken, Phys. Rev. D 27, 140 (1983).

[16] N.D. Birrell, P.C.W. Davies, Quantum Fields in Curved Space (Cambridge University Press, Cambridge, 1982).

[17] S. Jeon, T. Epelbaum, Annals of Physics 364, 1 (2016) arXiv:1506.00672].

[18] W.G. Unruh, Phys. Rev. D 14, 870 (1976).

[19] S. Takagi, Prog. Theor. Phys. Suppl. 88, 1 (1986).

[20] L.C.B. Crispino, A. Higuchi, G.E.A. Matsas, Rev. Mod. Phys. 80, 787 (2008) arXiv:0710.5373.

[21] N.B. Narozhny, A.M. Fedotov, B.M. Karnakov, V.D. Mur, V.A. Belinskii, Phys. Rev. D 65, $025004(2002)$. 
[22] S.A. Fulling, W. G. Unruh, Phys. Rev. D 70, 048701 (2004).

[23] N. B. Narozhny, A. M. Fedotov, B. M. Karnakov, V. D. Mur, V. A. Belinskii, Phys. Rev. D 70, 048702 (2004).

[24] D.C.M. Ostapchuk, S.Y. Lin, R.B. Mann, B.L. Hu, J. High Energ. Phys. (2012) 2012: 72. https://doi.org/10.1007/JHEP07(2012)072.

[25] J. Doukas, S.Y. Lin, B.L. Hu, R.B. Mann, J. High Energ. Phys. (2013) 2013: 119. https://doi.org/10.1007/JHEP11(2013)119.

[26] W.G. Unruh, R.M. Wald, Phys. Rev. D 29, 1047 (1984).

[27] S.J. Olson, T.C. Ralph, Phys. Rev. Lett. 106, 110404 (2011) arXiv:1003.0720].

[28] P. Martinetti, C. Rovelli, Class. Quant. Grav. 20, 4919 (2003) arXiv:gr-qc/0212074.

[29] D. Kharzeev and K. Tuchin, Nucl. Phys. A 753, 316 (2005).

[30] D. Kharzeev, Nucl. Phys. A 774, 315 (2006).

[31] D. Kharzeev, E. Levin, K. Tuchin, Phys. Rev. C 75, 044903 (2007).

[32] P. Castorina, D. Kharzeev, H. Satz, Eur. Phys. J. C 52, 187 (2007).

[33] F. Becattini, P. Castorina, J. Manninen, H. Satz, Eur. Phys. J. C 56, 493 (2008).

[34] P. Castorina, H. Satz, Adv. High Energy Phys. 2014, 376982 (2014).

[35] P. Castorina, A. Iorio, H. Satz, Int. J. Mod. Phys. E 24, 1550056 (2015).

[36] W.G. Unruh, N. Weiss, Phys. Rev. D 29, 1656 (1984).

[37] C.W. Bernard, Phys. Rev. D 9, 3312 (1974).

[38] N.P. Landsman, Ch.G. van Weert, Phys. Rep. 145, 141 (1987);

[39] J.I. Kapusta, C. Gale, Finite-Temperature Field Theory Principles and Applications (Cambridge University Press, Cambridge, 2006).

[40] F. Gelis, E. Iancu, J. Jalilian-Marian, R. Venugopalan, Ann. Rev. Nucl. Part. Sci. 60, 463 (2010) arXiv:1002.0333.

[41] Jean-Paul Blaizot, Rep. Prog. Phys. 80, 032301 (2017) arXiv:1607.04448.

[42] T. Lappi and L. McLerran, Nucl. Phys. A 772, 200 (2006) arXiv:hep-ph/0602189.

[43] R. Baier, A.H. Mueller, D. Schiff , D.T. Son, Phys. Lett. B 502, 51 (2001) arXiv:hep-ph/0009237.

[44] C. Shen, Nucl. Phys. A 956, 184 (2016) arXiv:1601.02563].

[45] J. Berges, K. Reygers, N. Tanji, R. Venugopalan, Nucl. Phys. A 967, 708 (2017) arXiv:1704.04032]. 